\title{
The fine guidance system of the PLATO mission
}

Denis Grießbach, Ulrike Witteck, Carsten Paproth

Denis Grießbach, Ulrike Witteck, Carsten Paproth, "The fine guidance system of the PLATO mission," Proc. SPIE 11852, International Conference on Space Optics - ICSO 2020, 118523H (11 June 2021); doi: 10.1117/12.2599604

SPIE Event: International Conference on Space Optics - ICSO 2021, 2021, Online Only 


\section{International Conference on Space Optics-ICSO 2020}

Virtual Conference

30 March-2 April 2021

Edited by Bruno Cugny, Zoran Sodnik, and Nikos Karafolas
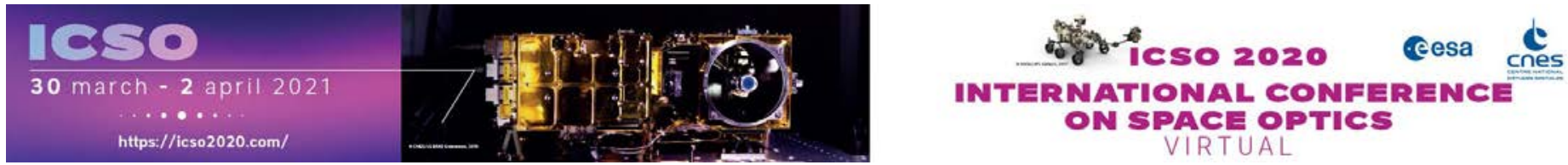

\section{The fine guidance system of the PLATO mission}

\section{Cesa isopueatans lecnes}

International Conference on Space Optics - ICSO 2020, edited by Bruno Cugny, Zoran Sodnik, Nikos Karafolas, Proc. of SPIE Vol. 11852, 118523H · (c) 2021 ESA and CNES

CCC code: $0277-786 \mathrm{X} / 21 / \$ 21 \cdot$ doi: $10.1117 / 12.2599604$ 


\title{
The Fine Guidance System of the PLATO Mission
}

\author{
Denis Grießbach ${ }^{\mathrm{a}}$, Ulrike Witteck ${ }^{\mathrm{b}}$, and Carsten Paproth ${ }^{\mathrm{c}}$ \\ ${ }^{\mathrm{a}, \mathrm{b}, \mathrm{c}}$ German Aerospace Center (DLR), Rutherfordstr. 2, 12489 Berlin, Germany
}

\begin{abstract}
PLATO - PLAnetary Transits and Oscillation of stars - is a medium-class mission in the European Space Agency (ESA) Cosmic Vision programme, whose launch is foreseen by 2026. The objective is the detection and characterization of terrestrial exoplanets up to the habitable zone of solar-type stars by means of their transit signature in front of a very large sample of bright stars. The seismic oscillations of the parent stars orbited by these planets is measured in order to understand the properties of the exoplanatory systems.

The PLATO payload consists of an instrument with 26 cameras for star observation; 24 normal cameras grouped in four subsets with six cameras each and two fast cameras. Besides providing scientific data for very bright stars, the fast cameras also serve as two redundant Fine Guidance System (FGS) and will be an integral part of the Attitude and Orbit Control System (AOCS). This ensures a very high pointing precision which is needed to achieve a high photometric precision.

Working as a star-tracker, the attitude calculation is based on guide star positions on the focal plane and their reference directions given by a star catalogue. Compared to predecessor missions like CoRoT, Kepler, or TESS, the precision of the fine guidance algorithm needs to be increased significantly. This is especially challenging as the optical design is identical for all cameras and optimized to meet the science objectives rather than to serve as a star-tracker. Therefore, a novel approach based on a Gaussian fit is proposed. The shown algorithm provides a noise optimal estimation of the guide star positions which propagates to an optimal attitude estimation. Although, computational more expensive than conventional methods, its suitability for a real-time on-board application is proven with an implementation on the target hardware. Furthermore, its robustness and precision is assessed theoretically and with simulated star images sequences.
\end{abstract}

Keywords: Star-Tracker, Navigation, Sensor

\section{INTRODUCTION}

The PLATO Mission will detect and characterize exoplanets by means of their transit signature in front of a very large sample of bright stars, and measure the seismic oscillations of the parent stars orbited by these planets in order to understand the properties of the exoplanatory systems. ${ }^{1}$ The nominal science operations consist of two phases. During the long-duration observation phase, two fields shall be observed for at least 2 years (up to 4 years) each. During the Step\&Stare observation phase, between 3 and 6 fields shall be observed for $2-5$ months, when feasible due to the position of the Sun.

The payload consists of an instrument with 26 telescopes for star observation; 24 normal telescopes and two fast telescopes. The fast telescopes have two system level tasks: First, they provide photometry

Further author information: (Send correspondence to Denis Grießbach)

Denis Grießbach: E-mail: denis.griessbach@dlr.de, Telephone: +49 (0)30 67055363 
for bright stars with magnitudes below 8.2. For that, the exposure time shall be sufficiently low to avoid a saturation of the detector. This is the main driver for the $2.5 \mathrm{~s}$ fast camera cycle time. Second, the fast cameras serve as two independent and redundant FGS to provide precise attitude measurements to the Spacecraft (S/C), making them an integral part of the AOCS Fine Pointing Mode (FPM). As the generic S/C AOCS capabilities are usually not compliant to the stringent pointing requirements of an exoplanet mission, this approach has also been used in missions like CoRoT, ${ }^{2}$ Kepler, ${ }^{3}$ and TESS. ${ }^{4}$

Table 1 illustrates the challenge faced by the PLATO FGS. It shows the required Noise Equivalent Angle (NEA), a measure of the FGS random noise, as well as the constraints given by the instrument. PLATO, like TESS, covers a very wide Field of View (FoV). With a limited number of pixels, this means a large instantaneous Field of View (iFoV), the spatial resolution of a single pixel. On the other hand the NEA is comparable to the Kepler mission providing a much smaller iFoV. With 1/50 pixel, the NEA requirement in terms of pixel resolution is quite similar for Kepler and TESS. However, for PLATO, the combination of a large FoV with strict pointing requirements leads to a demanding NEA requirement of about $1 / 600$ pixel. Furthermore, the optical design aims for a small Point Spread Function (PSF) which reduces the number of measurements for each guide star used within the FGS.

Table 1: Instrument Constraints and FGS Requirements

\begin{tabular}{|c|c|c|c|c|c|}
\hline & & CoRoT & Kepler & TESS & PLATO \\
\hline FoV [deg] & & $2.7 \times 3.05$ & $4 \times \pm 0.27$ & $24 \times 24$ & \pm 18.8 \\
\hline $\mathrm{iFoV}[\operatorname{arcsec}]$ & & 2.32 & 1.92 & 21 & 15 \\
\hline PSF size [pixel] & & $7 \times 7$ & $6.4 \times 6.4$ & $4 \times 4$ & $2 \times 2$ \\
\hline \# stars & & 2 & 40 & 200 & 30 \\
\hline \multirow{2}{*}{ NEA @ $2 \sigma[\operatorname{arcsec}]$} & $\mathrm{x}, \mathrm{y}$ & 0.3 & 0.04 & 0.4 & 0.025 \\
\hline & Z & 10 & - & 2.8 & 0.1 \\
\hline
\end{tabular}

To accommodate the given constraints and requirements a novel approach based on a Gaussian fit is proposed. The shown centroid algorithm provides a noise optimal estimation of the guide star positions which propagates to an optimal attitude estimation.

\subsection{Instrument}

The payload consists of 24 normal cameras and two fast cameras mounted on a highly stable optical bench. The normal cameras with a cadence of $25 \mathrm{~s}$ are grouped in four subsets with six telescopes. As shown in fig. 1b the four groups are tilted to cover a larger FoV. The fast cameras feature have a cadence of $2.5 \mathrm{~s}$ for the observation of very bright targets. Furthermore, they feature spectral filters (blue/red) to provide colour information for stellar analysis.

All cameras have the same optical design and contain four CCDs to cover the FoV. Each CCD has a sensitive area of $81.18 \mathrm{~mm} \times 81.18 \mathrm{~mm}$, equivalent to 4510 pixel $\times 4510$ pixel with a pixel size of $18 \mu \mathrm{m}$. With an $\mathrm{iFoV}$ of 15 arcsec, a single camera covers a FoV of $\pm 18.9^{\circ}$. To achieve the high cadence of the fast cameras, the CCDs are operated in frame transfer mode. Due to a metallization layer, as seen in fig. 2a, the light sensitive area for a frame transfer CCD is 4490 pixel $\times 2245$ pixel. Light collected on the sensitive area is shifted within $2 \mathrm{~s}$ to the read-out area. Here it is continuously read out by the Front End Electronics (FEE) and processed by the Digital Processing Unit (DPU). The FGS algorithm is running on a Leon2-FT processor at $80 \mathrm{MHz}$ and needs to be highly optimized to process 30 guide stars in less than $300 \mathrm{~ms}$. This is important to reduce latency which directly impacts the AOCS. 


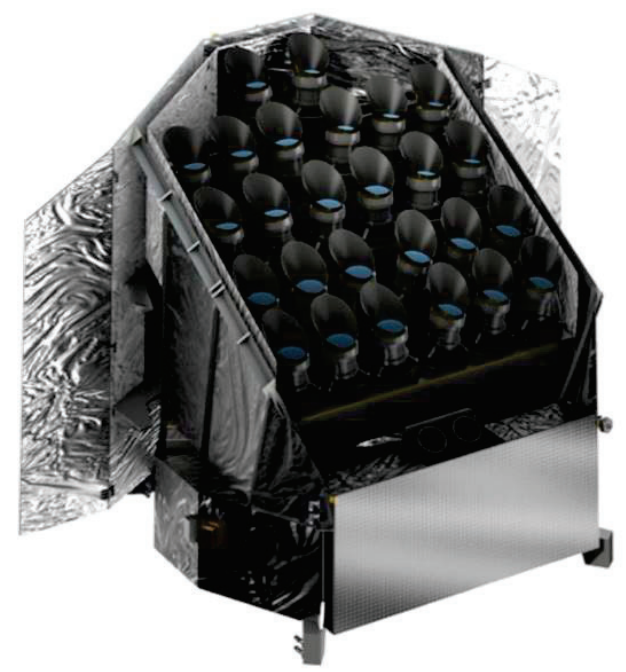

(a) Satellite (OHB Systems AG)

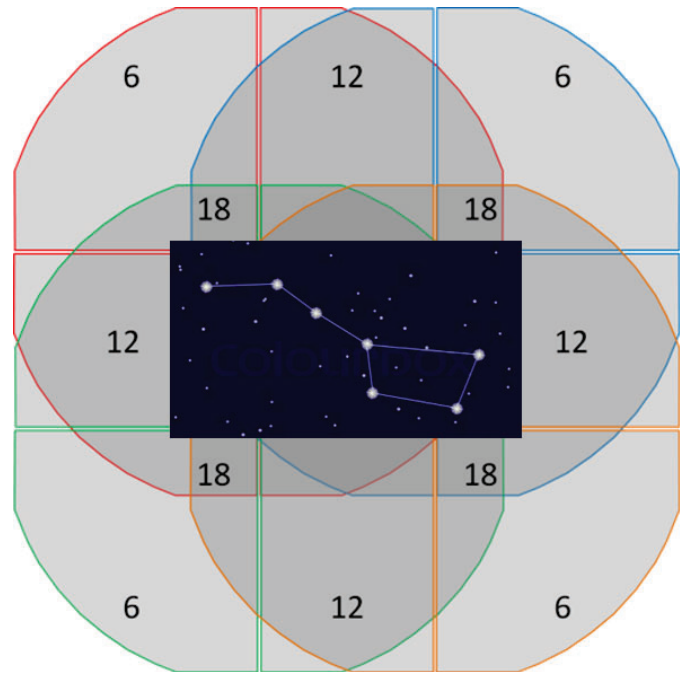

(b) Field of View

Figure 1: PLATO Instrument

\subsection{Camera Model}

A geometric camera model, sometimes referred to as FoV model, is needed to transform sky coordinates given in the camera boresight ${ }^{5}$ reference frame to the CCD reference frame and vice versa. It contains information regarding the Telescope Optical Unit (TOU), namely the focal length and a distortion model. Furthermore the position and rotation of the particular CCDs on the Focal Plane Assembly (FPA) w.r.t. the optical axis is determined. The different CCD reference frames are shown in fig. 2b.

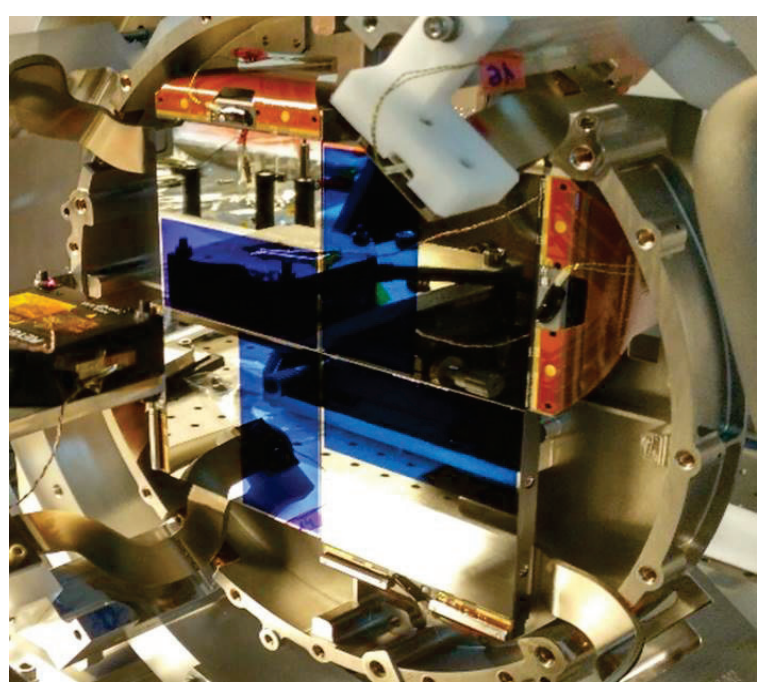

(a) FPA Prototype

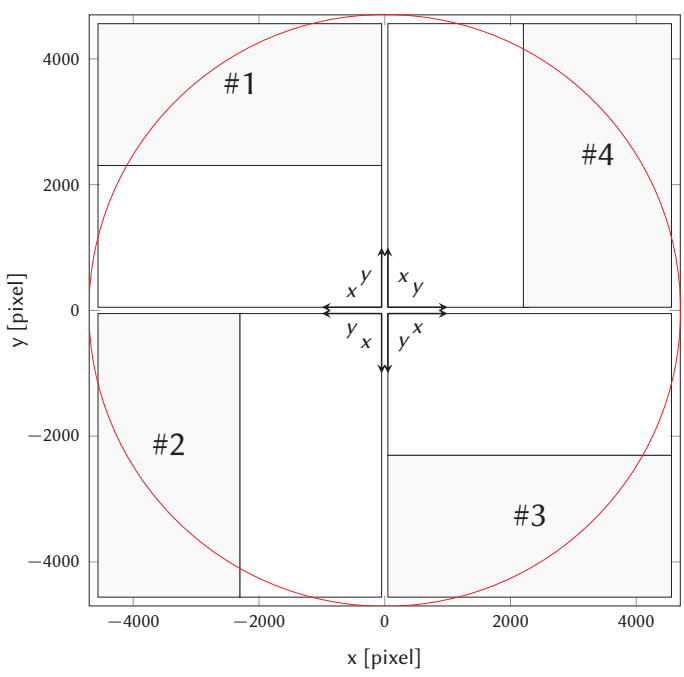

(b) FPA Layout

Figure 2: Frame Transfer CCDs 
Each camera model will be calibrated on-ground by using a collimated light source in combination with a hexapod to realize different field angles. This geometric calibration will be repeated on-board using dedicated stars.

\section{FINE GUIDANCE SYSTEM}

Working as a star tracker, the attitude calculation is based on guide star positions on the CCDs compared to their reference directions given by the star catalogue. Figure 3 shows an overview of the FGS algorithm providing quaternion measurements to the AOCS. As shown in section 2.1, each guide star centroid is estimated separately using a Gaussian PSF observation model. According to section 1.2, the centroid position given in its individual CCD frame is then transformed to a vector observation in the boresight reference frame for further processing. With at least two guide stars the attitude of the camera boresight frame w.r.t. the inertial reference frame can now be estimated by means of the QUaternion ESTimator (QUEST) algorithm, described in section 2.2.

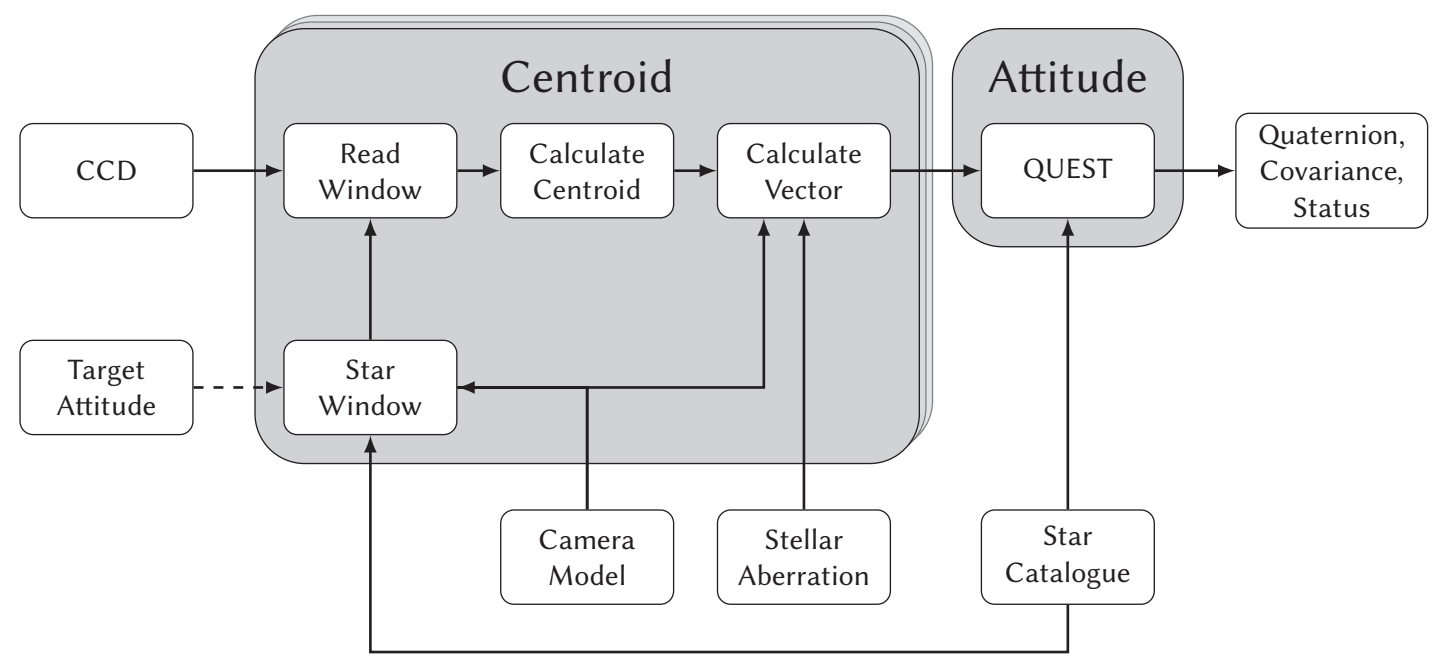

Figure 3: FGS algorithm overview

\subsection{Centroid Calculation}

The current state of the art algorithm to estimate the position or centroid of a guide star is the Centre of Mass (CoM) algorithm. It calculates the raw image moments

$$
\boldsymbol{M}_{p q}=\sum_{i} \sum_{j} i^{p} j^{q} \boldsymbol{y}(i, j)
$$

with the intensity of a particular pixel $\boldsymbol{y}(i, j)$. The centroid $(\bar{i}, \bar{j})^{T}=\left(\boldsymbol{M}_{10} / \boldsymbol{M}_{00}, \boldsymbol{M}_{01} / \boldsymbol{M}_{00}\right)^{T}$ is defined as a weighted mean over all pixels. This algorithm is fast, robust, and easy to implement but also has some major drawbacks. It does not handle possible background signals and is not optimal when subjected to random noise. Supposed the size of the star is considerably smaller than the window, the local background can be estimated by averaging the faintest pixels in the window, e.g. using the 20 border pixels of a $6 \times 6$ pixel window. 
The better choice in terms of random noise is to use a PSF fitting algorithm. Note that there is no substantial improvement in terms of non-linearity. An observation $\boldsymbol{h}(i, j)$ of a single pixel is modelled as a Gaussian PSF which we define as an integral over the area of a pixel of a Gaussian distribution.

$$
\boldsymbol{h}(i, j)=I_{m} \cdot \frac{1}{2 \pi \sigma^{2}} \int_{i}^{i+1} e^{-\frac{\left(u-u_{c}\right)^{2}}{2 \sigma^{2}}} \mathrm{~d} u \int_{j}^{j+1} e^{-\frac{\left(v-v_{c}\right)^{2}}{2 \sigma^{2}}} \mathrm{~d} v+D+\xi
$$

with the centroid position $\left(u_{c}, v_{c}\right)^{T}$, PSF width $\sigma$, intensity $I_{m}$, background $D$, and random noise $\xi$. The intensity of each star is computed with

$$
I_{m}=F_{m} t / g
$$

with the measured flux $F_{m}$ in $e^{-} / s$, the exposure time $t$ and a gain factor $g$. The flux depends on the star magnitude $m$, the effective light-collecting area $A$, the transmission of the optical system $T$, the quantum efficiency of the detector $Q$, and the reference flux $F_{0}$ of a star with $m=0$.

$$
F_{m}=F_{0} T Q A \cdot 10^{-0.4 m}
$$

The centroid of the PSF is estimated by minimizing the following non-linear functional

$$
\boldsymbol{r}(\boldsymbol{\alpha})=\min _{\boldsymbol{\alpha}}\|\boldsymbol{y}-\boldsymbol{h}\|_{2}
$$

with observations for each pixel $\boldsymbol{y}(i, j)$ and unknowns $\boldsymbol{\alpha}=\left(u_{c}, v_{c}, \sigma, D, I_{m}\right)^{T}$.

\subsection{Attitude Estimation}

After transforming the measured centroids to vector observations, the optimal attitude is estimated by means of the QUEST algorithm. ${ }^{6}$ With the star directions

$$
\hat{\boldsymbol{b}}=\frac{1}{\sqrt{\left(x^{2}+y^{2}+1\right)}}\left(\begin{array}{l}
x \\
y \\
1
\end{array}\right)
$$

given in the camera boresight reference frame and the corresponding reference unit vectors $\boldsymbol{r}_{i}$, given in an inertial reference frame, the following optimization problem arises.

$$
\mathcal{L}(\boldsymbol{A})=\frac{1}{2} \sum_{i=1}^{N} a_{i}\left\|\hat{\boldsymbol{b}}_{i}-\boldsymbol{A} \boldsymbol{r}_{i}\right\|^{2}
$$

Where $\boldsymbol{A}$ is an orthogonal matrix (direction-cosine matrix) and $a_{i}$ denoting non negative weights with $\sum_{i=1}^{N} a_{i}=1$ where $N$ is the number of measurements. For measurements with variance $\sigma^{2}, a_{i}=\sigma_{\text {tot }}^{2} / \sigma_{i}^{2}$ with $1 / \sigma_{\text {tot }}^{2}=\sum_{i=1}^{N} 1 / \sigma_{i}^{2}$. This optimization problem is often referred to as Wahba's problem. An efficient solution is the QUEST algorithm, which solves for the optimal quaternion minimizing eq. (7).

A possible drawback of the QUEST algorithm is a singularity which occurs if the scalar part of the quaternion equals zero. This can be avoided e.g. by applying an a priori quaternion known from the nominal pointing before estimation. Because an a priory reference frame rotation is assumed to be known, the QUEST method can be used without risk to run into singular cases. In case of an unknown orientation, the methods ESOQ (Estimator of the Optimal Quaternion) and ESOQ2 (Second Estimator of the Optimal Quaternion) provide build in mechanisms to avoid the singular case not needing explicit reference frame rotations. In terms of speed, QUEST is superior to Davenports q-method and about equal to ESOQ, ESOQ2, and FOAM. ${ }^{7}$ 


\section{EXPERIMENTAL RESULTS}

In order to prove the performance, robustness, and speed of the proposed algorithm, a prototype implementation has been developed and evaluated. Since there is no real data, the expected PLATO mission observations needs to be simulated as shown in section 3.1. The created image time series are fed into the processing pipeline to study the resulting attitude measurement as well as the intermediate results on centroid level.

\subsection{Simulation}

The PLATO simulator $\left(\right.$ PlatoSim $\left.^{8}\right)$ is a software tool designed to simulate image time series. It models a CCD and its read-out electronic including an exhaustive list of common noise sources, e.g. read-out noise, shot noise, dark noise, open shutter smearing, and cosmic rays to name just a few.

Figure 4 shows the used open loop test set-up, consisting of the simulated camera, S/C AOCS, and the FGS itself. For now the AOCS assumes a worst case FGS noise and simulates an $8 \mathrm{~Hz}$ time series of the predicted S/C movement, which is fed to the camera simulator. In a later stage of the development the loop will be closed by feeding the FGS measurement back to the AOCS.

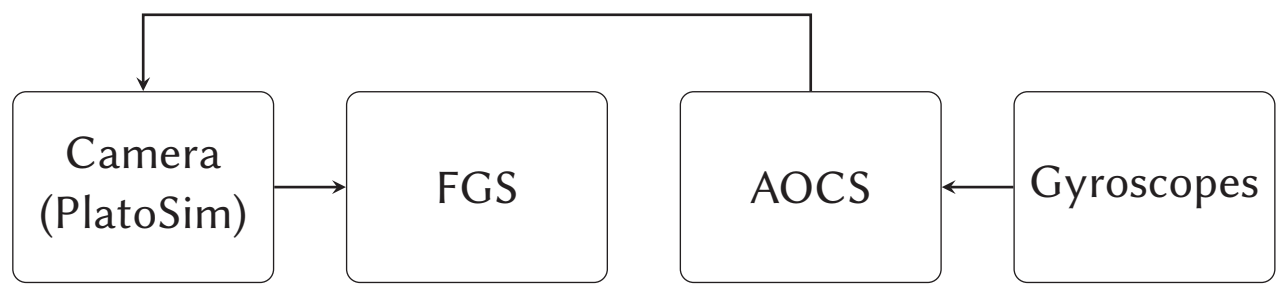

Figure 4: Open loop configuration

Furthermore, the telescope optics can be specified in terms of geometry, transmission, and PSF by means of a Zemax simulation. Figure 5a shows a typical input PSF for a particular field position. PlatoSim also takes CCD charge diffusion, a potentially diffusion of charge into neighbour pixels, into account to produce a system PSF as seen in fig. 5b. After placing the high resolution PSF to its projected position on the CCD it is sampled to its final resolution, shown in fig. 5c.

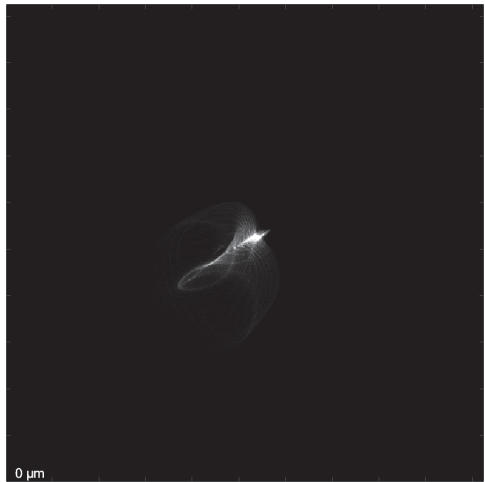

(a) Original Zemax PSF

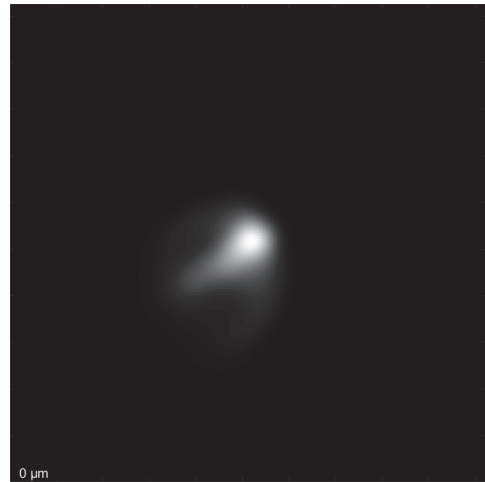

(b) High Resolution System PSF

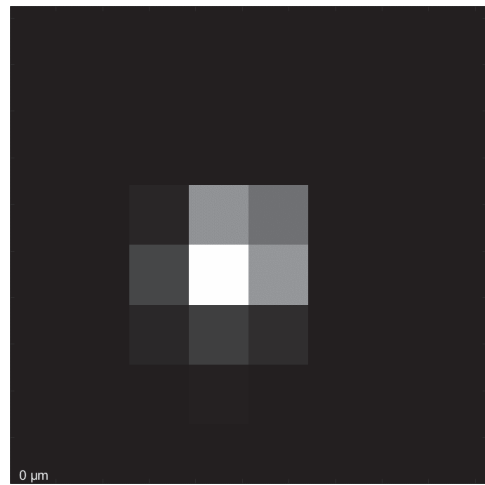

(c) Low Resolution System PSF

Figure 5: PlatoSim PSF Evolution 


\subsection{Performance Evaluation}

The overall performance of the FGS can be quantified with two error terms. First, the Noise Equivalent Angle (NEA), describing short term random noise and second, bias-stability which is long term systematic noise due to non-linearities within the centroid estimation. Bias-stability, caused by Thermo-Elastic Distortion (TED) and stellar aberration, has been studied but will not be the focus of this paper. Besides the number and distribution of the chosen guide stars, the centroid precision is the main factor for the expected performance in terms of NEA.

In order to get representative results 134 guide stars have been chosen for the first stellar field to be observed. Guide stars need to be in the right magnitude range $(5-7)$, must have low contamination from neighbouring stars, and low stellar variability. Due to the use of frame transfer CCDs only 80 of the chosen guide stars fall on the sensitive area of the CCDs. The baseline is to use 30 stars for the FGS algorithm.

\subsubsection{Centroid Precision}

The residual centroid error is calculated as difference between nominal star position - including averaged $\mathrm{S} / \mathrm{C}$ jitter - and the estimated centroid. Figure 6 shows the measured centroid noise $\sigma_{x, y}$ as well as an uncertainty estimate $\sigma_{x, y}^{\prime}$ derived from the expected read-out noise and shot noise. Since this estimate does not include the influence of S/C jitter, it can be seen as a lower bound, mainly dependent on the star magnitude which determines the Signal to Noise Ratio (SNR).

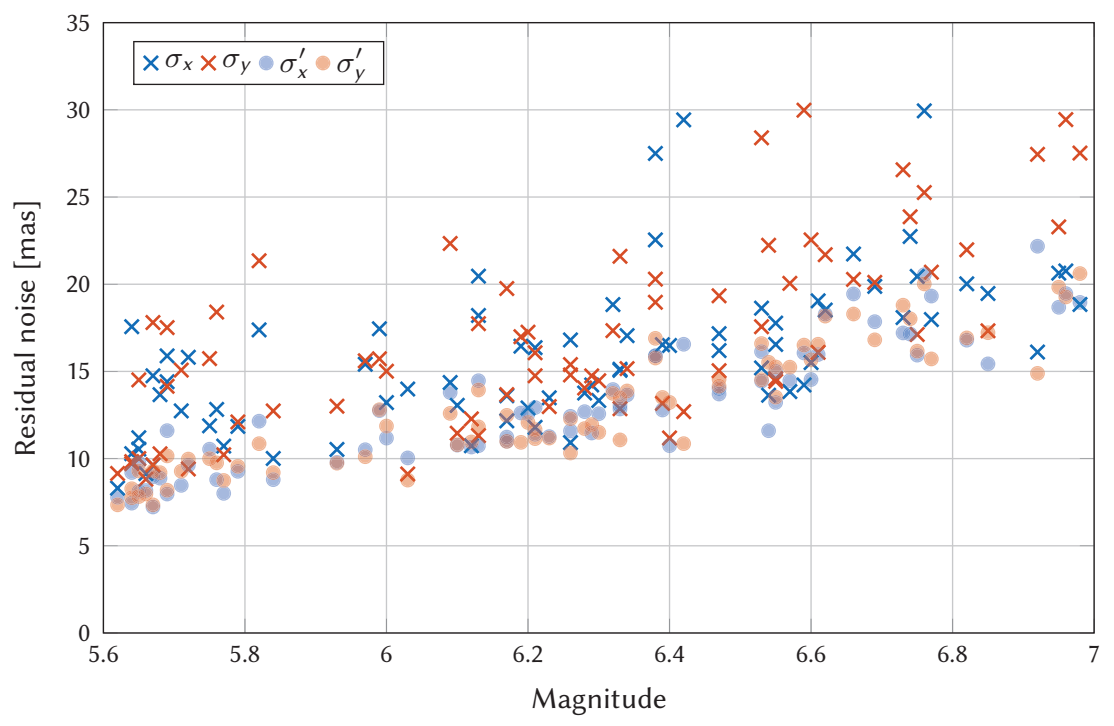

Figure 6: Residual centroid noise for blue non-Gaussian PSF

Besides the random noise sources, read-out noise and shot noise, an important driver for the centroid precision is the PSF shape which determines the sensitivity against S/C jitter. As seen in fig. 5, the PSF is rather non-Gaussian and varies widely over the FoV. This effect is amplified if local PSF features, e.g. minima or maxima, are located near a pixel edge. Small movements induced by jitter will lead to a disproportionately strong change in pixel illumination which results in additional noise for the estimated centroid position. In case of a Gaussian PSF shape this would be covered by the observation model given in eq. (2). 
Table 2: Non-Gaussian PSF: mean residual noise [mas]

\begin{tabular}{lcccc}
\hline & \multicolumn{2}{c}{ CoM } & \multicolumn{2}{c}{ Gauss-fit } \\
\cline { 2 - 5 } & $\left\langle\sigma_{x}\right\rangle$ & $\left\langle\sigma_{y}\right\rangle$ & $\left\langle\sigma_{x}\right\rangle$ & $\left\langle\sigma_{y}\right\rangle$ \\
\hline blue & 31.4 & 31.9 & 15.9 & 16.7 \\
red & 31.4 & 31.9 & 16.6 & 17.5 \\
\hline
\end{tabular}

Table 2 shows the mean residual noise for the centroid positions using the CoM method as well as the introduced Gaussian fit for a window size of $6 \times 6$ pixel. Here, the performance of the Gaussian fit is improved by a factor 2 compared to the traditional CoM algorithm. This means a factor 4 less guide stars are needed to achieve a similar FGS performance. Since we are dealing with a limited amount of very bright targets, this already is a significant improvement. Note that the CoM precision is reduced with larger windows, e.g. 44 mas for $7 \times 7$ pixel windows. This is not the case for the Gaussian fit algorithm which further increases its advantage.

\subsubsection{Attitude Precision}

The NEA does not only depend on the individual noise at centroids level but also on the number and distribution of the used guide stars. A rough estimation is made by averaging the mean centroid noise $\epsilon_{\text {centroid }}$ with the number of stars $N_{\text {stars }} .{ }^{9}$ The directional error is given as follows:

$$
\epsilon_{x y}=\frac{\epsilon_{\text {centroid }}}{\sqrt{N_{\text {stars }}}} .
$$

Assuming uniformly distributed stars across a rectangular FoV, the roll-axis precision can be estimated with

$$
\epsilon_{z}=\frac{\epsilon_{\text {centroid }}}{\sqrt{N_{\text {stars }}}} \frac{\sqrt{6}}{\beta_{F o V}},
$$

where $\beta_{F o V}=36$ deg is the FoV angle. For 30 stars with a mean directional centroid precision of 17 mas as given in table 2, the directional FGS precision is 3.1 mas, respectively 12.1 mas for the roll axis. Due to the geometry of the frame transfer CCDs yielding a reduced FoV, the roll axis performance will be decreased.

The influence regarding selection and distribution of guide stars has been studied by setting up a Monte Carlo simulation. The outcome is a mean error with an uncertainty rather than a single result holding only for a particular set of guide stars. As shown in section 3.2, 80 guide stars are available for the given pointing direction in the simulation. Randomly chosen 30 guide stars are used to calculate the attitude as shown in section 2.2. With the simulation input jitter serving as a ground truth the residual FGS error can be calculated. Because the selection of guide stars is the same as in table 2, the FGS performance shown in table 3 nicely follows the estimation given in eqs. (8) and (9).

Table 3: Mean FGS NEA for different PSFs [mas]

\begin{tabular}{lccc}
\hline & $\sigma_{x}$ & $\sigma_{y}$ & $\sigma_{z}$ \\
\hline blue & $3.1 \pm 0.3$ & $2.9 \pm 0.3$ & $16.5 \pm 1.8$ \\
red & $3.0 \pm 0.3$ & $3.2 \pm 0.4$ & $14.7 \pm 1.4$ \\
\hline
\end{tabular}


To take possible guide star selections and distributions into account, the associated requirements are given with a $95 \%$ Confidence Interval (CI) with ensemble statistical interpretation. The FGS performance is therefore calculated as given in table 4. It shows that the NEA requirement of 25 mas for transverse axes and 100 mas for the rotational axis is fulfilled in all cases including maturity margin.

Table 4: Mean FGS NEA at $95 \%$ CI [mas]

\begin{tabular}{lccc}
\hline & $\sigma_{x}$ & $\sigma_{y}$ & $\sigma_{z}$ \\
\hline blue & 7.2 & 6.8 & 40.1 \\
red & 7.1 & 8.0 & 34.8 \\
\hline
\end{tabular}

\section{CONCLUSION}

In this paper, the PLATO Fine Guidance System has been presented. The instrument itself works as a star tracker and is used as integral part of the S/C AOCS to provide very precise attitude measurements.

The main challenge for the PLATO FGS is to meet very strict pointing requirements with a very wide FoV camera. To compensate for the low spatial resolution of the instrument, a novel Gaussian fit algorithm was developed. It provides an optimal estimate of the guide star positions and successfully suppresses random noise by a factor $2-3$ compared to the traditional CoM method. Furthermore, the instrument is designed to provide a very high SNR which, together with the improved algorithm, leads to a significantly increased performance in comparison to predecessor missions.

In order to assess the performance of the FGS a complex simulation chain has been established to produce image time series. A prototypical implementation was used to evaluate the simulated data and finally predict the expected FGS performance. Although this paper focuses on the assessment of the NEA, bias-stability being low frequent noise caused by TED and stellar aberration, has been studied as well and will be subject of a follow-up publication.

As shown in many simulation runs, the presented algorithm is compliant to the NEA requirement as well as to all requirements regarding bias-stability and timing. The timing was measured with a prototypical implementation on the target hardware. In the course of the following phases the FGS evaluation will be repeated with further refined simulations and consolidated S/C jitter time series. The next step will be the finalization of the Engineering Model (EM) Application Software (ASW) featuring a functional FGS. This includes extensive testing to ensure a seamless integration for the first closed loop simulation efforts.

\section{ACKNOWLEDGMENTS}

This work has benefited from financial support by DLR in the framework of its contribution to the PLATO Mission. It presents results from the European Space Agency (ESA) space mission PLATO. PLATO data is being processed by the PLATO Mission Consortium (PMC). Funding for the PMC is provided by national institutions, in particular the institutions participating in the PLATO MultiLateral Agreement (MLA). The PLATO mission website is www.cosmos.esa.int/plato and the PMC website is https://platomission.com/. 


\section{REFERENCES}

[1] H. Rauer, C. Catala, C. Aerts, T. Appourchaux, W. Benz, A. Brandeker, J. Christensen-Dalsgaard, M. Deleuil, L. Gizon, M.-J. Goupil, and et al., "The plato 2.0 mission," Experimental Astronomy 38, p. 249-330, Sep 2014.

[2] M. Auvergne, P. Bodin, L. Boisnard, J.-T. Buey, S. Chaintreuil, G. Epstein, M. Jouret, T. Lam-Trong, P. Levacher, A. Magnan, and et al., "The CoRoT satellite in flight: description and performance," Astronomy Es Astrophysics 506, p. 411-424, Mar 2009.

[3] D. Putnam, C. Schira, and S. C. McQuerry, "Kepler Fine Guidance Sensor Operation," in ADVANCES IN THE ASTRONOMICAL SCIENCES, Rocky Mountain Guidance and Control Conference, 137, pp. 289-308, Published for the American Astronautical Society by Univelt;, (San Diego, Calif.), 2010.

[4] T. Nguyen, E. Morgan, R. Vanderspek, A. Levine, M. Kephart, J. Francis, J. Zapetis, K. Cahoy, and G. R. Jr., "Fine-pointing performance and corresponding photometric precision of the Transiting Exoplanet Survey Satellite," Journal of Astronomical Telescopes, Instruments, and Systems 4(4), pp. 1-13, 2018.

[5] E. C. for Space Standardization, Stars sensors terminology and performance specification, no. ECSS-E-ST60-20C, ESA Publications Division, Nov. 2008.

[6] M. D. Shuster, "Approximate algorithms for fast optimal attitude computation," in AIAA Guidance and Control Conference, 1978.

[7] Y. Cheng and M. D. Shuster, "The speed of attitude estimation," Journal of the Astronautical Sciences 127, pp. 101-116, 2007.

[8] Marcos-Arenal, P., Zima, W., De Ridder, J., Aerts, C., Huygen, R., Samadi, R., Green, J., Piotto, G., Salmon, S., Catala, C., and Rauer, H., "The plato simulator: modelling of high-precision high-cadence space-based imaging," A 3 A 566, p. A92, 2014.

[9] F. L. Markley and J. L. Crassidis, Fundamentals of Spacecraft Attitude Determination and Control, Space Technology Library, Springer, New York, 2014. 